01

\title{
Особенности затухания свободной поляризации в ультратонких газовых ячейках
}

\author{
(C) А.Ч. Измайлов \\ Институт фризики НАН Азербайджана, \\ AZ-1143 Баку, Азербайджан \\ e-mail: azizm57@rambler.ru
}

Поступила в редакцию 06.02.2020 г.

В окончательной редакции 06.02.2020 г.

Принята к публикации 02.04.2020 г.

\begin{abstract}
Теоретически исследован оптический эффект затухания свободной поляризации в ультратонкой газовой ячейке, внутренняя толщина которой меньше или порядка длины волны возбуждающего монохроматического лазерного импульса, пропускаемого ортогонально плоскопараллельным стенкам данной ячейки. Установлен новый механизм исследуемого эффекта, обусловленный спецификой рассогласования фаз светоиндуцированных атомных дипольных моментов вследствие пролетной релаксации атомов в такой ячейке. В результате этого динамика затухания свободной поляризации в рассматриваемой ситуации радикально отличается от известного случая в обычной (макроскопической) газовой ячейки. Обнаружены и проанализированы нетривиальные осцилляционные зависимости сигналов затухания свободной поляризации от отношения толщины подобной ультратонкой ячейки к длине волны излучения возбуждающего импульса.
\end{abstract}

Ключевые слова: затухание свободной поляризации, ультратонкая газовая ячейка, когерентное излучение, фазы дипольных моментов.

DOI: $10.21883 /$ OS.2020.08.49699.39-20

\section{Введение}

Сигнал затухания свободной поляризации (ЗСП) обусловлен когерентным излучением, испускаемыми атомами (молекулами) среды после того, как они возбуждены импульсом лазерного излучения [1,2]. Действительно, такой импульс, резонансный некоторому атомному переходу $a \leftrightarrow b$, создает суперпозицию квантовых уровней $a$ и $b$. В результате в среде образуется совокупность осциллирующих со временем дипольных моментов. После выключения исходного лазерного импульса такие атомные диполи продолжают некоторое время колебаться и излучать регистрируемый сигнал ЗСП. Данный когерентный сигнал распространяется в направлении возбуждающего импульса, поскольку только в этом направлении излучение этих диполей оказывается сфазированным. Вследствие поступательного движения атомов (молекул) газовой среды происходит рассогласование по фазе их дипольных моментов, что и является причиной наблюдаемого сигнала ЗСП.

В обычной макроскопической ячейке с газовой средой характерное время ЗСП, индуцируемого лазерным импульсом, весьма мало, так как определяется величиной, обратной допплеровскому уширению спектральной линии резонансного оптического перехода [1,2]. Вместе с тем к настоящему времени изготавливаются и используются в спектроскопии ультратонкие газовые ячейки с характерной внутренней толщиной $l$ вплоть до десятков нанометров [3]. В частности, при лазерном зондировании атомов в таких ячейках зарегистрированы внутридопплеровские резонансы поглощения, структура которых существенно зависит от отношения толщины $l$ к длине волны $\lambda \sim 1 \mu \mathrm{m}$ облучаемого света [4]. Это обусловлено спецификой индуцирования и разрушения оптической когерентности атомов при их пролете между плоскопараллельными стенками данных ячеек. В настоящей работе показано, что при возбуждении атомов (молекул) газовой среды в ультратонкой ячейке достаточно коротким импульсом когерентного монохроматического излучения подобные процессы приводят к нетривиальным сигналам ЗСП, которые также в значительной степени определяются отношением $l / \lambda$.

\section{Основные соотношения}

Рассмотрим разреженную газовую среду между плоскопараллельными прозрачными стенками одномерной ячейки с внутренней толщиной $l$. Этот газ полагается настолько разреженным, что взаимодействием между его атомами (молекулами) можно пренебречь. Ортогонально стенкам ячейки вдоль оси $z$ через газовую среду в течение времени $\tau$ проходит импульс монохроматического когерентного излучения, электрическая компонента которого имеет вид

$$
\mathscr{E}(z, t)=0.5 \mathbf{e} E(t) \exp [i(\omega t-k z)]+c . c .,
$$

где $E$ и $\mathbf{e}-$ соответственно амплитуда и единичный вектор поляризации излучения, $\omega-$ его частота, $k=2 \pi / \lambda-$ волновое число, $\lambda-$ длина волны, c.c. - комплексно сопряженная величина. Амплитуда $E(t)$ этого импульса отлична от 0 лишь в промежутке 
времени $t$ от $(-\tau)$ до 0 . Частота излучения $\omega \gg \tau^{-1}$ близка к центру $\omega_{0}$ перехода $a \leftrightarrow b$ между основным $a$ и возбужденным $b$ атомными уровнями. Длительность $\tau$ импульса (1) полагается настолько короткой, что выполняются следующие соотношения:

$$
k u \tau \ll 1, \quad\left|\omega-\omega_{0}\right| \tau \ll 1, \quad u \tau \ll l,
$$

где $u$ - наиболее вероятная скорость атомов в газе, $k u$ - допплеровское уширение спектральной линии, которое гораздо больше константы радиационного распада $\gamma$ оптической когерентности на переходе $a \leftrightarrow b$. При условиях (2) в течение данного импульса все атомы газовой среды можно рассматривать как резонансные [1,2], пренебрегая радиационной релаксацией возбужденных атомов, а также количеством этих атомов, которые успевают столкнуться со стенками ячейки. Тогда в процессе такого короткого импульса можно написать следующую систему уравнений для элемента матрицы плотности оптической когерентности $\rho_{a b}$, а также относительных населенностей $\rho_{a}$ и $\rho_{b}$ уровней резонансного перехода $a \leftrightarrow b$ с матричным элементом $d$ дипольного момента [2]:

$$
\begin{gathered}
\rho_{a}+\rho_{b}=1, \\
\frac{d \rho_{a b}}{d t}=0.5 i g(t)\left(\rho_{b}-\rho_{a}\right), \\
\frac{d \rho_{b}}{d t}=i g(t) \rho_{a b},
\end{gathered}
$$

где величину $g(t)=E(t) d / \hbar$ можно считать действительной для рассматриваемой задачи. Из уравнений (3) получаем известное выражение $[1,2]$ для оптической когерентности $\rho_{a b}^{(0)}$ в момент времени $t=0$ прекращения импульса (1):

$$
\rho_{a b}^{(0)}=0.5 i \sin \left[\int_{-\tau}^{0} g(t) d t\right]=0.5 i \sin \left[(d / \hbar) \int_{-\tau}^{0} E(t) d t\right]
$$

Изменение оптической когерентности $\rho_{a b}$ в течение последующего времени $t>0$ описывается следующим уравнением для атомов с проекцией скорости $v$ вдоль оси $z$ [2]:

$$
\frac{\partial \rho_{a b}}{\partial t}+v \frac{\partial \rho_{a b}}{\partial z}+\Lambda \rho_{a b}=0
$$

где

$$
\Lambda=\gamma+i(\delta-k v),
$$

$\delta=\left(\omega-\omega_{0}\right)-$ отстройка частоты $\omega$ излучения (1) от центральной частоты $\omega_{0}$ резонансного перехода $a \leftrightarrow b$. Решение уравнения (5) должно удовлетворять начальному условию:

$$
\rho_{a b}(z, t=0)=\rho_{a b}^{(0)},
$$

где величина $\rho_{a b}^{(0)}$ определена в (4). Кроме того, функция $\rho_{a b}(z, t)$ должна удовлетворять начальным условиям, которые зависят от особенностей столкновений атомов (молекул) со стенками рассматриваемой ячейки. Как и в предыдущих теоретических исследованиях для тонких газовых ячеек $[4,5]$, будем считать, что в результате каждого из таких столкновений происходит полное разрушение оптической когерентности. Тогда из уравнения (5) получаем следующее решение для атомов среды между стенками ячейки с координатами $z=0$ и $z=l$ :

$$
\begin{gathered}
\rho_{a b}(z, v, t)=\rho_{a b}^{(0)} \exp (-\Lambda t) \eta(z-v t) \eta(l-z+v t), \\
(t \geq 0),
\end{gathered}
$$

где $\eta(x)$ - ступенчатая функция $(\eta(x)=1$, если $x \geq 0$ и $\eta(x)=0$, когда $x<0)$.

Импульсное излучение (1) индуцирует поляризацию газовой среды следующего вида $[1,2]$ :

$$
\mathbf{Q}(z, t)=0.5 \mathbf{e} P(z, t) \exp [i(\omega t-k z)]+c . c .,
$$

где

$$
P(z, t)=2 N d \int_{-\infty}^{\infty} \rho_{a b}(z, v, t) F(v) d v
$$

$N$ - плотность атомов, $\rho_{a b}-$ рассчитанный выше элемент матрицы плотности оптической когерентности, $F(v)$ - распределение Максвелла по проекции атомной скорости $v$ на ось $z$ :

$$
F(v)=\pi^{-0.5} u^{-1} \exp \left(-v^{2} u^{2}\right) .
$$

Несмотря на прерывание возбуждающего импульса (1) в момент $t=0$, индуцируемая им поляризация среды $(9),(10)$ является при $t>0$ источником когерентного сигнала ЗСП, электрическая компонента которого имеет вид $[1,2]$

$$
\mathscr{E}_{S}(z, t)=0.5 \mathbf{e} E_{S}(z, t) \exp [i(\omega t-k z)]+c . c .
$$

На основе уравнений Максвелла [2] устанавливается связь амплитуды $E_{s}$ сигнала ЗСП (12) на выходе из ячейки с соответствующей компонентой поляризации среды (9), (10):

$$
\begin{aligned}
E_{S}(l, t) & =-2 i \pi k \int_{0}^{l} P(z, t) d z \\
& =-4 i \pi N k d \int_{-\infty}^{\infty} F(v)\left[\int_{0}^{l} \rho_{a b}(z, v, t) d z\right] d v,
\end{aligned}
$$

где $k=\omega / c . \quad$ Подставляя в $\rho_{a b}(z, v, t)(8)$, при $t \geq 0$ получаем выражение

$$
E_{s}(l, t)=-4 i \pi N d \rho_{a b}^{(0)}(k l) W(l, t) \exp (-i \delta t),
$$

где

$$
W(l, t)=\exp (-\gamma t) \int_{-l / t}^{l / t} F(v) \exp (i k v t)\left(1-\frac{|v|}{l} t\right) d v .
$$


Множитель $\exp (-i \delta t)$ в (14) указывает на то, что атомы излучают сигнал ЗСП (12) на частоте $\omega_{0}$ резонансного перехода $a \leftrightarrow b$. Заметим, что в известном случае обычной (макроскопической) газовой ячейки $[1,2]$ вместо функции $W(l, t)(15)$ в выражении (14) будет стоять следующая величина:

$$
\begin{aligned}
W_{0}(t) & =\exp (-\gamma t) \int_{-\infty}^{\infty} F(v) \exp (i k v t) d v \\
& =\exp \left[-\gamma t-0.25(k u t)^{2}\right] .
\end{aligned}
$$

Тогда интенсивность соответствующего сигнала ЗСП будет пропорциональна $l^{2}$, и, согласно (16), данный сигнал может быть существенным лишь весьма короткое время порядка $2(k u)^{-1}$. В следующем разделе будут установлены принципиально новые особенности продолжительности и энергии сигнала ЗСП для ультратонких газовых ячеек.

\section{Обсуждение результатов}

На эксперименте может быть зарегистрирована интенсивность сигнала ЗСП, которая пропорциональна величине $\left|E_{s}(l, t)\right|^{2}(14)$. Поэтому затухание такого сигнала определяется зависимостью $W(l, t)(15)$ от времени $t$, представленной на рис. 1 для различных значений толщины $l$ ультратонкой ячейки в случае допплеровски уширенной спектральной линии резонансного перехода $a \leftrightarrow b$ с однородной полушириной $\gamma=0.01 k u$. На рис. 1, $a$ показана также зависимость $W_{0}(t)(16)$ для сигнала ЗСП в обычной (макроскопической) ячейке. Видно, что данный сигнал фактически не проявляется уже при $t>5(k u)^{-1}$ в отличие от гораздо более медленного ЗСП в ультратонких газовых ячейках. В области $t>5(k u)^{-1}$ ярко проявляется зависимость ЗСП от отношения $l / \lambda$ толщины $l$ ячейки к длине волны $\lambda$ когерентного излучения (рис. $1, b)$. Так, через время $t \gg l / u$ функция $W(l, t)(15)$ имеет вид

$$
W(l, t) \approx \frac{2[1-\cos (k l)]}{\pi^{0.5} k^{2} u l} \frac{\exp (-\gamma t)}{t} .
$$

Согласно (17), динамика ЗСП в значительной степени определяется фактором $k l=2 \pi(l / \lambda)$. Действительно, рассогласование фаз излучения атомных диполей через время $t>0$ после прекращения действия возбуждающего импульса (1) описывается величиной $k v t$ для атомов с различными проекциями скорости $v$. На это указывает множитель $\exp (i k v t)$ в (15) и (16). Вследствие столкновительной релаксации на стенках ультратонкой ячейки через время $t>0$ оптическая когерентность сохраняется лишь у атомов с проекциями скорости $|v| \leq l / t$, для которых разброс фаз $k|v| t \leq k l$. Поэтому на рис. 1 наблюдается существенная зависимость динамики ЗСП от отношения $l / \lambda$.
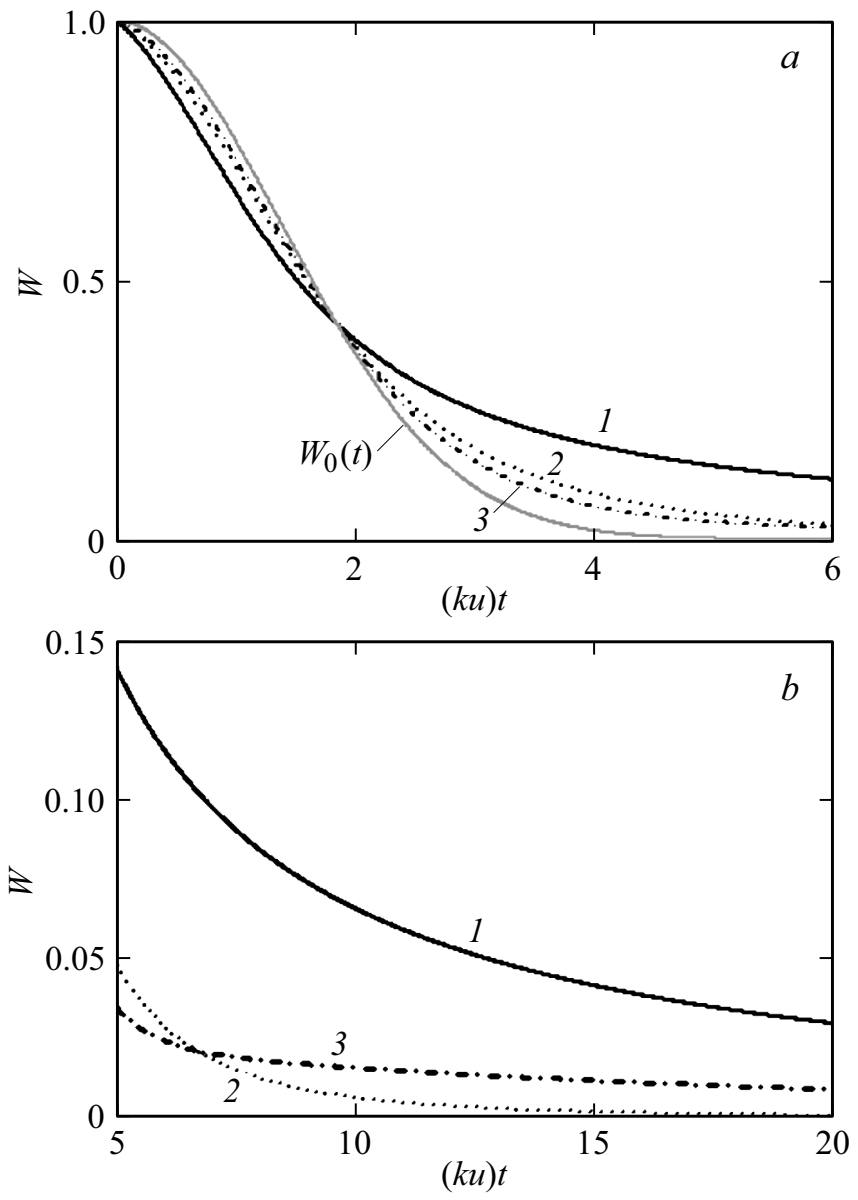

Рис. 1. Зависимости величины $W(l, t)$ от времени $t$ (в единицах $k^{-1} u^{-1}$ ) для значений $l / \lambda=0.5(1), 1(2), 1.5$ (3) при $\gamma /(k u)=0.01$. Кривая $W_{0}(t)$ на рис. $1, a$ соответствует случаю обычной макроскопической ячейки. Рисунок $1, b$ представляет данные зависимости в области $t \geq 5(k u)^{-1}$.

На эксперименте может быть измерена также энергия сигнала ЗСП за время его регистрации, начиная с некоторого момента времени $\Delta>0$. Такая энергетическая характеристика описывается следующей величиной:

$$
R(l, \Delta)=\int_{\Delta}^{\infty}\left|E_{s}(l, t)\right|^{2} d t,
$$

где интенсивность сигнала $\left|E_{s}(l, t)\right|^{2}$ определяется из (14). Согласно рис. 1 , существенная зависимость сигнала ЗСП от толщины ячейки $l$ проявляется при $t>2(k u)^{-1}$. Поэтому на рис. 2 представлены зависимости величины $R(18)$ от $l$ при различных значениях момента $\Delta \geq 3(k u)^{-1}$ начала регистрации сигнала ЗСП. Виден осцилляционный характер зависимости энергии ЗСП от толщины $l$, который наиболее ярко проявляется при $l \leq u \Delta$. Тогда в соответствии с формулой (17) энергия ЗСП достигает максимумов при толщине ячейки $l=(n+0.5) \lambda$ и минимумов, если $l=n \lambda$ для целых чисел $n=0,1,2, \ldots$ (кривые 2 и 3 на рис. 2 ). С ростом 


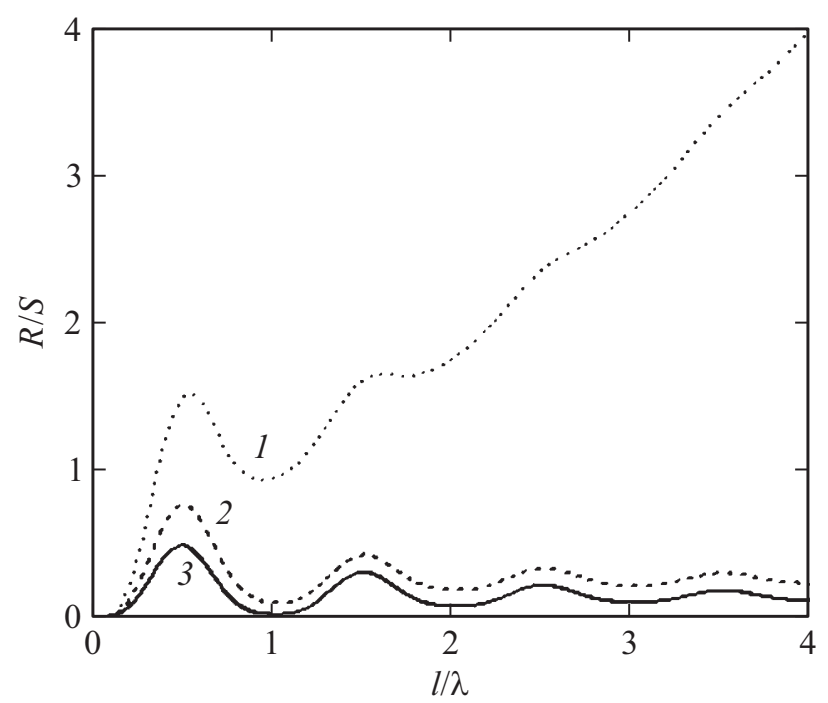

Рис. 2. Зависимости энергетической характеристики $R$ сигнала ЗСП (в единицах $S=16 \pi^{2} N^{2}|d|^{2}\left|\rho_{a b}^{(0)}\right|^{2} k^{-1} u^{-1}$ ) от толщины ячейки $l$ (в единицах $\lambda$ ) для различных моментов времени $\Delta$ с начала регистрации $\Delta(k u)=3$ (1), 5 (2) и 7 (3) при $\gamma /(k u)=0.01$.

толщины $l$ амплитуда таких осцилляций в зависимости $R(l)$ уменьшается.

Таким образом, в настоящей работе установлены нетривиальные зависимости ЗСП от отношения $l / \lambda$ толщины $l$ ультратонкой газовой ячейки к длине волны $\lambda$ когерентного излучения. Сигнал ЗСП возникает уже после прохождения достаточно интенсивного возбуждающего лазерного импульса через резонансную газовую среду. Поэтому, несмотря на сравнительную слабость такого сигнала, полученные в настоящей работе зависимости ЗСП от времени и толщины ультратонкой ячейки могут быть зарегистрированы на эксперименте.

\section{Список литературы}

[1] Laser and Coherence Spectroscopy / Ed. by Steinfeld J.S. N. Y:: Plenum Press, 1978. 529 р. Перевод: Лазерная и когерентная спектроскопия/Под ред. Стейнфелда Дж. М.: Мир, 1982. 629 с.

[2] Акулин В.М., Карлов Н.В. Интенсивные резонансные взаимодействия в квантовой электронике. М.: Наука, 1987. 312 c.; Akulin V.M., Karlov N.V. Resonant Interactions in Quantum Electronics. Berlin: Springer-Verlag, 1992. 300 p.

[3] Peyrot T., Sortais Y.R.P., Greffet J.-J., Browaeys A., Sargsyan A., Keaveney J., Hughes I.G., Adams C.S. // Phys. Rev. Lett. 2019. V. 122. N 11. P. 113401.

[4] Dutier G., Yarovitski A., Saltiel S., Papoyan A., Sarkisan D., Bloch D., Ducloy M. // Europhys. Lett. 2003. V. 63. N 1. P. 35.

[5] Измайлов А.Ч. // Опт. и спектр. 2019. Т. 126. В. 6. С. 702; Izmailov A.Ch. // Opt. Spectrosc. 2019. V. 126. N 6. P. 624. 